\title{
Characteristics and outcome of long-stay patients in a paediatric intensive care unit in Cape Town, South Africa
}

\author{
T L Nupen, ${ }^{1}$ FCPaed (SA); A C Argent, ${ }^{1,2}$ MD, FCPaed (SA); B M Morrow, ${ }^{1} \mathrm{PhD}$ \\ ${ }^{1}$ Department of Paediatrics and Child Health, Faculty of Health Sciences, University of Cape Town, South Africa \\ ${ }^{2}$ Paediatric Intensive Care Unit, Red Cross War Memorial Children's Hospital, Cape Town, South Africa
}

Corresponding author: B M Morrow (brenda.morrow@uct.ac.za)

\begin{abstract}
Background. Paediatric intensive care is a costly, specialised and limited resource in low- and middle-income countries. The implications of extended paediatric intensive care unit (PICU) stay in South Africa (SA) are not known.

Objectives. To describe the characteristics, outcomes and resource consumption of long-stay patients (LSPs) and to identify predictive factors for long PICU stay.

Methods. A retrospective review of routinely collected data on all children admitted to an SA PICU over one calendar year. Long PICU stay was defined statistically as $>19$ days. Long- and short-stay patient (SSP) groups were compared, and variables significantly associated with long stay on univariate analysis were entered into a stepwise multiple regression model.

Results. Over the study period, 1126 children (median age 8 months, 60.9\% male) were admitted to the PICU, occupying 5936 bed-days; 54 LSPs (4.8\%) utilised 1807 (30.4\%) bed-days. Mortality and the standardised mortality ratio (actual/mean predicted mortality) in LSPs and SSPs were $29.6 \%$ v. $12 \%(p=0.002)$ and 2.4 v. $0.7(p=0.002)$, respectively. Median duration of stay for LSPs and SSPs was 29.5 days and 2 days, respectively ( $p<0.0001)$. LSPs were younger than SSPs (median 4 months (interquartile range $2-17)$ v. 9 months $(2-34) ; p=0.03$ ), and fewer were male ( $48 \%$ v. $61.6 \%, p=0.049)$. On multivariate analysis, only female gender was independently associated with long PICU stay. Conclusions. LSPs represent a small proportion of PICU admissions, yet have a higher mortality rate than SSPs and consume disproportionate PICU resources. No predictive model could be established for early recognition of potential LSPs to plan PICU bed allocation effectively.
\end{abstract}

S Afr Med J 2017;107(1):70-75. DOI:10.7196/SAMJ.2017.v107i1.11279

Paediatric intensive care is a costly, specialised and limited resource that should be used as effectively and efficiently as possible. Paediatric intensive care unit (PICU) costs can range from about USD70 to over >USD1 000 per patient day, depending on the sophistication and complexity of services offered. ${ }^{[1]}$ In the context of low- and middle-income countries (LMICs), where there is often limited access to PICU care, it is particularly important to ensure that resources are used rationally, while upholding the ethical principle of distributive justice. Rational PICU use includes careful monitoring of patient outcomes relative to resource utilisation and applying appropriate admission criteria to contain financial, staffing and social costs. ${ }^{[2]}$

Intensive care is now offered to children with complex and chronic conditions who may not have been admitted to PICUs in the past. ${ }^{[3]}$ Advances in PICU care have resulted in increased survival of critically ill patients, some of whom require long-term PICU stay. Compared with short-stay patients (SSPs), long-stay patients (LSPs) require a different spectrum of resource allocation, with greater involvement of the extended multidisciplinary team, to improve their PICU experience and optimise their functional outcomes. This constitutes a challenge in terms of staffing needs, costs, and the PICU system, structure and function. ${ }^{[4]}$

It has been suggested that patients who have a long duration of PICU stay use a disproportionate amount of resources compared with SSPs. Together with the perceived vulnerability of LSPs to increased mortality and morbidity, there is a concern about appropriate resource allocation to this group. There is a paucity of outcome data for LSPs, especially from Africa. Existing outcome data from other settings describe a wide spectrum of LSPs, with outcomes ranging from excellent to poor. ${ }^{[3,5]}$ On an individual level, the expected outcome for a child with Guillaine-Barré syndrome, for example, will differ from that of a child with end-stage Duchenne's muscular dystrophy, although both may be LSPs in the PICU.

While it is accepted that length of stay (LOS) may reflect severity of illness and PICU quality and performance, there is no uniform definition of what constitutes long PICU stay. Previous studies have defined LSPs as having a PICU LOS of anywhere beyond 7 days to $>30$ days ${ }^{[6-8]}$ with various methods used to identify these thresholds.

It is useful to describe the characteristics, impact and outcomes of LSPs in order to plan their care more economically, ensure optimal involvement of the multidisciplinary team, optimise patient outcome, enable appropriate counselling of family members, and develop guidelines for limitation or withdrawal of medical care. ${ }^{[2]}$ Most existing literature on LSPs in PICUs report findings from developed countries, which may have different population and burden of disease profiles from developing countries.

\section{Objectives}

To determine an appropriate threshold for defining long PICU stay in a South African (SA) PICU, to describe the characteristics and outcomes of these LSPs, and to determine any predictive factors associated with long PICU stay.

\section{Methods \\ Study design and setting}

The setting was the 22-bed multidisciplinary PICU at Red Cross War Memorial Children's Hospital, Cape Town, SA. This PICU admits $\sim 1400$ children per annum, of whom $\sim 500$ are emergency 
admissions, mostly for the management of infectious diseases, and the majority require invasive mechanical ventilation. Extracorporeal membrane circulation was not available at the time of the study. PICU beds are in great demand and there is pressure to maintain rapid patient turnover. Long-term invasive and non-invasive ventilation is available for stable patients not requiring PICU-level care in other wards in the hospital.

This was a retrospective descriptive study of all children admitted to the PICU over one calendar year. Clinical data were extracted from a pre-existing PICU database.

Permission to conduct the study was obtained from the institutional human research ethics committee (ref. no. 105/2011), and the need for informed consent was waived owing to the retrospective nature of the study.

\section{Statistical analysis}

The definition of long PICU LOS in our setting was modelled using the following techniques, described by Weissman: ${ }^{[7]}$ (i) for normally distributed data, two standard deviations (SDs) above the mean LOS; (ii) five times the median LOS; (iii) beyond the 75th and 95\% percentile of the median LOS; and (iv) visual examination of the frequency distribution graph of LOS v. number of admissions. A specific LOS was identified from the start of the 'tail' of the distribution curve (if not normally distributed). The bestfit model for our data was then chosen to distinguish between LSPs and SSPs.

Data were tested for normality using the Shapiro-Wilks test. Data were not normally distributed and are therefore presented throughout this article as medians (interquartile ranges, IQRs) for continuous data and proportions for categorical data. The characteristics and outcomes of the long-stay group were compared with those of the short-stay group using the Mann-Whitney $U$-test and the $\chi^{2}$ or Yates corrected $\chi^{2}$ test as appropriate.

Variables significantly associated with the binary outcome of interest (LSPs) on univariate analysis were entered into a forward, stepwise, logistic regression model to determine independent predictive factors for long stay, with the intention of developing a clinical predictive algorithm.

The proportion of ICU days consumed by LSPs was calculated to determine resource allocation.

Statistical analyses were conducted using Statistica version 11 (StatSoft, USA) and a significance level of $p<0.05$ was chosen.

\section{Results}

Over the study period, 1126 children (median age 8 months (IQR 2 32 ), $60.9 \%$ male), occupying 5936 bed days, were admitted to the PICU. Baseline patient data are presented in Table 1.

\section{Defining the LSP}

Considering that the data were not normally distributed, it was not considered appropriate to identify long stay as two SDs above the mean LOS. ${ }^{[7]}$

The model using the $>75$ th percentile of the median LOS corresponded to PICU LOS of 6 days. Two hundred and fifty-three patients $(22.5 \%)$ fulfilled this criterion, with a median LOS of 11 days (IQR 8 - 17) and a $14.0 \%$ mortality rate.

When using five times the median PICU LOS ( $>15$ days) for identification of LSPs, 76 (6.7\%) of patients fulfilled the definition, with a median LOS of 25.5 days (IQR 18.5 - 35.5) and $30.0 \%$ mortality.

Modelling long stay using the $>95$ th percentile of the median LOS was found to be equivalent to the visual examination of the start of the 'tail' of the distribution curve (Fig. 1), with 54 patients (4.8\%) spending $\geq 20$ days in the PICU, with a median LOS of 29.5 days (IQR 25 - 40) and a $29.6 \%$ mortality rate.

We therefore chose to define LSPs as those having a duration of stay of $>19$ days according to the two best-fit models.

\section{LSP characteristics and outcomes}

In comparison with SSPs, LSPs were significantly younger, and greater proportions were female and admitted for emergency care. The main reason for admission of LSPs was management of paediatric illness, including sepsis, pneumonia and congenital heart disease, whereas SSPs were more commonly admitted for postoperative care, traumarelated injury and poisoning (Tables 1 and 2).

LSPs had significantly higher mortality and a significantly higher standardised mortality ratio (actual/mean predicted mortality using the Paediatric Index of Mortality Score version 2 (PIM2) compared with SSPs (Table 1). There were no significant differences between LSPs who died and those who survived (Table 3).

In the final multiple regression model, only female gender was independently associated with the outcome of long stay, making it impossible to develop a predictive model for LSPs (Table 4).

\section{Resource utilisation}

LSPs comprised $4.8 \%$ of the PICU population yet utilised $30.4 \%$ (1 807) of the total PICU bed days during the study period (Fig. 2).

\section{Discussion}

This study presents data from an SA setting that support international reports that LSPs are a small but consistent proportion of the PICU population who utilise substantial PICU resources yet have worse outcomes than other PICU patients. ${ }^{[2,3,6]}$

Although LSPs are increasingly acknowledged as an integral part of PICU planning and care, they have been a difficult group to prospectively identify and investigate ${ }^{[6]}$ mainly owing to the lack of a uniform definition of what constitutes an LSP. A particular LOS may be designated as a threshold to identify the LSP, but this threshold varies widely in the literature. ${ }^{[3,6-8]}$ We established a rational basis for defining long PICU stay in our context, and identified significant differences between our short- and long-stay populations.

Similar to previous studies, ${ }^{[6,9]}$ we used the $>95$ th percentile of the median PICU LOS to define LSPs, which also corresponded to the start of the tail of the frequency distribution curve. This duration of $>19$ days was also reported by Jeena et al. ${ }^{[11]}$ in another SA PICU over

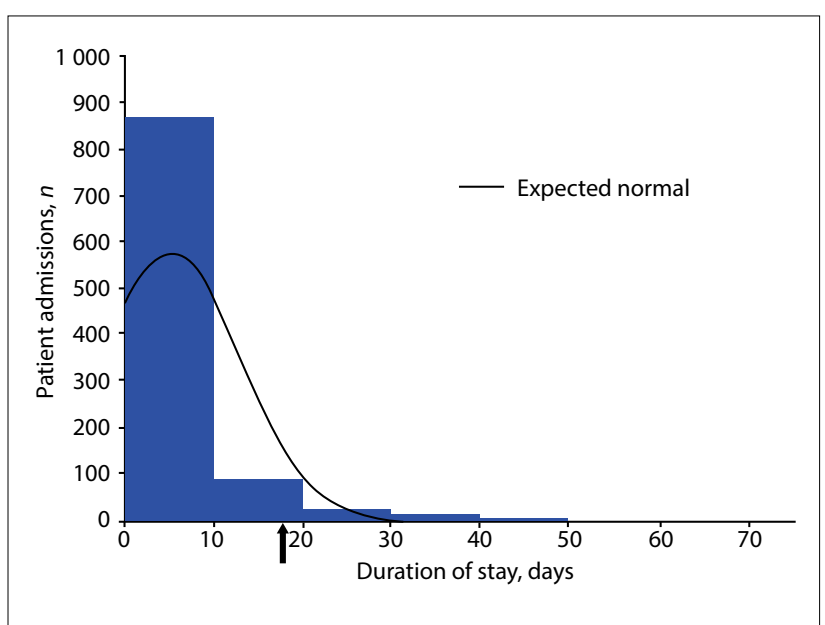

Fig. 1. LOS distribution curve. The arrow reflects the start of the visual tail and the 95th percentile. 
a decade ago, but is lower than that reported by other authors using different methods. ${ }^{[3,5,8,9,11]}$

It is important to standardise institutional definitions of the LSP in order to engage in ongoing audits and practise improvement initiatives; to plan the care of LSPs more economically, including counselling of families about additional risks and complications of an extended PICU stay; to motivate for involvement of the extended multidisciplinary team to provide holistic care to the child while he or she is in the PICU; and to ensure prompt recognition of medical futility. It would also be useful to have a uniform, global definition of an LSP for appropriate data comparison among PICUs. The definition delineated in this study could provide a rational comparison for further studies conducted in similar contexts around the world.

On univariate analysis, LSPs were found to be younger than SSPs, and a greater proportion were female and admitted for emergency reasons, similar to previous reports. ${ }^{[6]}$ Although lower admission weight was associated with long PICU stay, this was not adjusted for patient age or height and cannot therefore be interpreted as malnutrition, wasting or stunting. Capturing nutritional characteristics in future studies would be helpful, as these data reflect the health of the population and are potentially modifiable factors.

Previous studies from $\mathrm{SA}^{[10]}$ and India ${ }^{[12]}$ described most admission diagnoses in LSPs as being of infective origin, while studies from developed countries list congenital heart disease, neurological disease and metabolic disorders as the more common diagnoses in LSPs. ${ }^{[12]}$ Our LSP PICU admission diagnoses and profile were diverse owing to the varied case mix in our multidisciplinary setting. It was notable, however, that LSPs were diagnosed more frequently with pneumonia, congenital heart disease without surgery, and tracheo-oesophageal fistula or oesophageal atresia than the short-stay group. The high incidence of pneumonia is expected in the SA context of significant infectious disease burden. We did not record comorbid conditions other than HIV, as this is the most prevalent comorbidity in our population group. In future studies it would be useful to capture

Table 1. Admission characteristics and outcomes of all included patients, and comparison of long- and short-stay patient groups

\begin{tabular}{|c|c|c|c|c|}
\hline & $\begin{array}{l}\text { All } \\
(N=1126)\end{array}$ & $\begin{array}{l}\text { LSPs } \\
(n=54)\end{array}$ & $\begin{array}{l}\text { SSPs } \\
(n=1072)\end{array}$ & $p$-value \\
\hline Gender (males/females) & $\begin{array}{l}686: 440 \\
(60.9 \% \text { male })\end{array}$ & $\begin{array}{l}26: 28 \\
(48.1 \% \text { male })\end{array}$ & $\begin{array}{l}660: 412 \\
(61.6 \% \text { male })\end{array}$ & 0.049 \\
\hline Age (mo.), median (IQR) & $8(2-32)$ & $4(2-17)$ & $9(2-34)$ & 0.03 \\
\hline Weight (kg), median (IQR) & $7(3.6-13.2)$ & $4.75(3.1-10.2)$ & $7.15(3.6-13.6)$ & 0.04 \\
\hline Risk of mortality using PIM2 score & $0.0723(0.0259-0.1923)$ & $0.096(0.0487-0.1490)$ & $0.0712(0.0255-0.1938)$ & 0.4 \\
\hline Emergency admissions, $n$ (\%) & $832(73.9)$ & $46(85.2)$ & $786(73.3)$ & 0.05 \\
\hline \multicolumn{5}{|l|}{ Reason for admission, $n$ (\%) } \\
\hline Paediatric illness & $676(60.0)$ & $41(75.9)$ & $635(58.3)$ & 0.02 \\
\hline Poisoning & $15(1.3)$ & $0(0)$ & $15(1.4)$ & 0.8 \\
\hline Non-accidental injury & $4(0.4)$ & $1(1.9)$ & $3(0.3)$ & 0.5 \\
\hline Post cardiac surgery & $188(16.7)$ & $9(16.7)$ & $179(16.7)$ & 0.9 \\
\hline Post thoracic surgery & $16(1.4)$ & $0(0)$ & $16(1.5)$ & 0.8 \\
\hline Post abdominal surgery & $74(6.6)$ & $1(1.9)$ & $73(6.7)$ & 0.2 \\
\hline Post cranial surgery & $50(4.4)$ & $1(1.9)$ & $49(4.6)$ & 0.5 \\
\hline Post spinal surgery & $15(1.3)$ & $0(0)$ & $15(1.4)$ & 0.8 \\
\hline Post airway surgery & $16(1.4)$ & $0(0)$ & $16(1.5)$ & 0.8 \\
\hline Post surgery, other & $15(1.3)$ & $0(0)$ & $15(1.4)$ & 0.8 \\
\hline MVA & $26(2.3)$ & $0(0)$ & $26(2.4)$ & 0.5 \\
\hline Other accident & $31(2.8)$ & $1(1.9)$ & $30(2.8)$ & 0.99 \\
\hline \multicolumn{5}{|l|}{ HIV status } \\
\hline HIV test not done & $544(48.3)$ & $22(40.7)$ & $522(48.6)$ & 0.3 \\
\hline $\begin{array}{l}\text { HIV-positive, not symptomatic } \\
\text { of AIDS }\end{array}$ & $78(6.9)$ & $1(1.9)$ & $77(7.2)$ & 0.2 \\
\hline HIV-negative & $409(36.3)$ & $22(40.7)$ & $387(36.1)$ & 0.5 \\
\hline $\begin{array}{l}\text { HIV-positive and mildly } \\
\text { symptomatic }\end{array}$ & $95(8.4)$ & $7(13.0)$ & $88(8.2)$ & 0.3 \\
\hline Mortality, $n(\%)$ & $145(12.9)$ & $16(29.6)$ & $129(12.0)$ & 0.0002 \\
\hline $\begin{array}{l}\text { Risk-adjusted mortality (actual/mean } \\
\text { predicted) }\end{array}$ & 0.77 & 2.4 & 0.7 & 0.002 \\
\hline $\begin{array}{l}\text { Duration of PICU stay (days), median } \\
\text { (IQR) }\end{array}$ & $3.0(1-6)$ & $29.5(25-40)$ & $2(1-5)$ & $<0.0001$ \\
\hline
\end{tabular}


Table 2. Specific primary diagnoses under the admission diagnostic category 'paediatric illness'

\begin{tabular}{|c|c|c|c|}
\hline Specific primary diagnoses, $n(\%)$ & $\begin{array}{l}\text { LSPs } \\
(n=41)\end{array}$ & $\begin{array}{l}\text { SSPs } \\
(n=1072)\end{array}$ & $p$-value \\
\hline Sepsis (including meningococcal) & $2(4.9)$ & $54(5.0)$ & 0.75 \\
\hline Pneumonia/pneumonitis & $13(31.7)$ & $195(18.2)$ & 0.03 \\
\hline Cardiomyopathy/endo-/myo-/pericarditis & $3(7.3)$ & $24(2.2)$ & 0.1 \\
\hline Congenital heart disease without surgery & $13(31.7)$ & $58(5.4)$ & $<0.0001$ \\
\hline Necrotising enterocolitis & $1(2.4)$ & $19(1.8)$ & 0.8 \\
\hline Tracheo-oesophageal fistula or oesophageal atresia & $3(7.3)$ & $2(0.2)$ & $<0.0001$ \\
\hline Burns related & $1(2.4)$ & $7(0.7)$ & 0.7 \\
\hline Non-HIV immunodeficiency & $1(2.4)$ & $4(0.4)$ & 0.5 \\
\hline Chronic lung disease & $1(2.4)$ & $1(0.1)$ & 0.1 \\
\hline Other shock & $1(2.4)$ & $2(0.2)$ & 0.2 \\
\hline Congenital diaphragmatic hernia & $1(2.4)$ & $5(0.5)$ & 0.5 \\
\hline Rheumatic heart disease & $0(0)$ & $5(0.5)$ & 0.5 \\
\hline Gastroschisis & $0(0)$ & $4(0.4)$ & 0.3 \\
\hline Central nervous system & $0(0)$ & $62(5.8)$ & 0.2 \\
\hline Guillain-Barré syndrome & $0(0)$ & $2(0.2)$ & 0.1 \\
\hline Gastroenteritis & $0(0)$ & $61(5.7)$ & 0.2 \\
\hline Upper airway obstruction & $0(0)$ & $17(1.6)$ & 0.9 \\
\hline Bronchiolitis & $0(0)$ & $11(1.0)$ & 0.9 \\
\hline Apnoea & $0(0)$ & $11(1.0)$ & 0.9 \\
\hline Asthma & $0(0)$ & $5(0.45)$ & 0.5 \\
\hline Gastrointestinal tract & $0(0)$ & $21(2.0)$ & 0.7 \\
\hline Tuberculosis & $0(0)$ & $3(0.3)$ & 0.2 \\
\hline Renal failure & $0(0)$ & $5(0.5)$ & 0.5 \\
\hline Kwashiorkor & $0(0)$ & $2(0.2)$ & 0.1 \\
\hline Inhaled foreign body & $0(0)$ & $2(0.2)$ & 0.1 \\
\hline
\end{tabular}

all comorbid diseases and chronic care device use, as these may predispose to long PICU stay. ${ }^{[6]}$

Diagnosis-specific mortality rates may guide critical care pathways ${ }^{[6]}$ and admission policies. There is the perception that certain conditions, such as Guillain-Barré syndrome, require prolonged LOS but have an excellent outcome, while other conditions such as extensive burns or cardiomyopathy may also result in prolonged LOS but have a poorer prognosis. This requires further research to inform admission policies in individual units.

We used the PIM2 model for the prediction of mortality during the study period, which has been validated in this PICU. ${ }^{[13]}$ We found that although the admission PIM2 score was an excellent predictor of overall mortality $(p<0.0001)$, it was not a predictive factor for long PICU stay.

PICU LOS is a marker of severity of illness, resource utilisation and performance of a PICU. International studies have consistently described small PICU LSP groups (1 - 7.5\% of the total PICU population) consuming a disproportionately large amount of available resources $(18-50 \%){ }^{[3,6,8,11]}$ Our data support these findings, with LSPs constituting $<5 \%$ of the PICU population, yet calculated to have utilised $30.4 \%$ of the total PICU bed days throughout the study period, and having an almost three-fold risk of death compared with the SSP group. LSP mortality rates have previously been reported to range from two-fold to almost ten-fold higher than those of SSPs. ${ }^{[3,6,8,11]}$ The fact that the standardised mortality ratio was also significantly higher than that of SSPs suggests that the actual outcomes of LSPs were worse than admission predictions of outcome.

We were unable to identify any clinically relevant predictive factors associated with long PICU stay from admission data. It is suggested that in future prospective studies, patients be reassessed after $\geq 2$ weeks of PICU stay to attempt to identify factors predictive of outcome at that point. Once a patient has been recognised as being an LSP, it would be appropriate to discuss the ongoing care plan as a multidisciplinary team, including family counselling as appropriate. ${ }^{[1,14]}$ Ongoing assessment of the LSP would ensure timeous review of medical futility criteria in order to avoid prolonging suffering and ensure rational resource allocation.

An Australian study concluded that more than two-thirds of children who spent $>28$ days in a PICU had an unfavourable outcome (moderate disability, severe disability or death), with almost half the LSPs having died at 6 months' follow-up after PICU discharge. ${ }^{[3]}$ A Canadian prospective observational study found longer PICU stays to be independently associated with poor quality of life and functional outcome at follow-up after 1 month. ${ }^{[15]}$ PICU LOS is a treatment-related exposure that has been highlighted as a predictor of post-critical illness psychiatric morbidity, including post-traumatic stress disorder and depression. ${ }^{[16]}$ In our study, outcome was measured using PICU survival and non-survival only. Owing to the retrospective nature of this study, we were unable to measure quality of life or morbidity of survivors of long PICU stay, 
Table 3. Differences between LSPs who died and those who survived

\begin{tabular}{|c|c|c|c|}
\hline & Alive $(n=38)$ & Died $(n=16)$ & $p$-value \\
\hline Male gender, $n(\%)$ & $18(47.4)$ & $8(50.0)$ & 0.9 \\
\hline Age (mo.), median (IQR) & $4.5(2.0-17.0)$ & $3.5(2.0-15.0)$ & 0.9 \\
\hline Weight $(\mathrm{kg})$, median (IQR) & $4.8(3.1-10.2)$ & $4.9(3.3-10.0)$ & 0.9 \\
\hline Risk of mortality using PIM2 score & $0.099(0.056-0.141)$ & $0.090(0.023-0.254)$ & 0.8 \\
\hline Emergency admissions, $n$ (\%) & $34(89.5)$ & $12(75.0)$ & 0.3 \\
\hline \multicolumn{4}{|l|}{ Reason for admission, $n(\%)$} \\
\hline Paediatric illness & $30(78.9)$ & $11(68.8)$ & 0.7 \\
\hline Poisoning & 0 & 0 & - \\
\hline Non-accidental injury & $1(2.6)$ & 0 & 0.7 \\
\hline Post cardiac surgery & $5(13.2)$ & $4(25.0)$ & 0.5 \\
\hline Post thoracic surgery & 0 & 0 & - \\
\hline Post abdominal surgery & 0 & $1(6.3)$ & 0.7 \\
\hline Post cranial surgery & $1(2.6)$ & 0 & 0.7 \\
\hline Post spinal surgery & 0 & 0 & - \\
\hline Post airway surgery & 0 & 0 & - \\
\hline Post surgery, other & 0 & 0 & - \\
\hline MVA & 0 & 0 & - \\
\hline Other accident & $1(2.6)$ & 0 & 0.7 \\
\hline \multicolumn{4}{|l|}{ HIV status, $n(\%)$} \\
\hline Test not done & $17(44.7)$ & $5(31.3)$ & 0.5 \\
\hline HIV-positive, not symptomatic of AIDS & $1(2.6)$ & 0 & 0.7 \\
\hline HIV-negative & $14(36.8)$ & $8(50.0)$ & 0.6 \\
\hline HIV-positive and mildly symptomatic & $4(10.5)$ & $3(18.8)$ & 0.8 \\
\hline $\begin{array}{l}\text { Duration of ICU stay before discharge or death (d), } \\
\text { median (IQR) }\end{array}$ & $27(25-36)$ & $38.5(26.5-45.0)$ & 0.08 \\
\hline
\end{tabular}

Table 4. Final multivariate logistic regression model (adjusting for all variables shown) for the binary outcome of long stay

\begin{tabular}{lllll}
\hline & Adjusted odds ratio & 95\% confidence interval & $p$-value & 0.047 \\
Female gender & 1.7 & $1.001-3.03$ & 0.2 \\
Age & 0.99 & $0.98-1.01$ & 0.8 \\
Weight & 1.01 & $0.96-1.05$ & 0.3 \\
Paediatric illness as reason for admission & 1.5 & $0.71-3.33$ & 0.4 \\
Emergency admission & 1.46 & $0.60-3.55$ & & 0.4
\end{tabular}

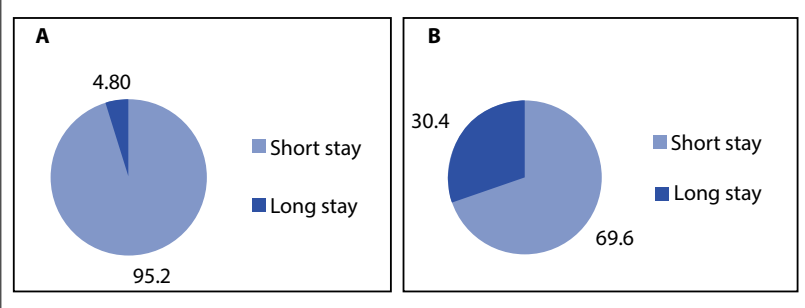

Fig. 2. (A) Proportion of LSPs in the PICU population, and (B) their resource consumption (in terms of bed utilisation) relative to SSPs.

and this constitutes a study limitation. We did not collect data related to previous PICU admissions and how this influenced subsequent outcome, and this is recommended for future studies.
This study did not address the nature of PICU care required by LSPs. In many cases the nursing, therapeutic input and psychosocial needs of these children and their families may differ profoundly from those of acutely ill SSPs. Parents too are at higher risk of adverse consequences of their child's admission. A prospective study in the USA highlighted frequent conflicts in the families of LSPs. ${ }^{[14]}$

Long-term functional outcome and quality of life measures may be more useful in the context of improved PICU survival, and it is recommended that these outcomes be used in future prospective studies of LSPs. ${ }^{[3]}$ There is much scope for future research on the quality of life of PICU survivors, particularly in the SA context where there is a low ratio of health professionals to patients, the social welfare disability grant offered to children with a moderate to severe disability is currently USD120 per month, and there 
is poor infrastructure and inaccessibility to transport in much of the country. ${ }^{[17]}$ Identifying whether high PICU mortality has been traded for a higher prevalence of morbidity has potential ramifications for provision and delivery of healthcare to this vulnerable population.

The poor outcome of LSPs reported here and in previous studies raises concerns around inappropriate resource consumption in the PICU. We recognise that in the SA setting, equitable distribution of healthcare services is an important ethical consideration when budgeting and planning for healthcare resource allocation.

\section{Conclusions}

It is important to standardise institutional definitions of LSPs. Our study defined LSPs as patients with a PICU LOS of $>19$ days based on the two best-fit models: >95th centile of duration of stay and the visual 'tail' of the LOS distribution curve. The definition delineated in this study could provide a rational basis for comparison between similar PICUs across the world.

LSPs represent a small percentage of PICU admissions yet have significantly increased mortality (greater than expected at the time of admission) and consume a disproportionate amount of resources compared with SSPs. This pattern from an SA setting is in keeping with international trends.

We were unable to establish a predictive model for the early recognition of potential LSPs in order to plan PICU bed allocation effectively.

Further investigations are needed to assess the quality of life of survivors of long PICU stay, particularly in LMICs.

1. Baker T. Critical care in low-income countries. Trop Med Int Health 2009;14(2):143-148. http://dx.doi. org/10.1111/j.1365-3156.2008.02202.
2. Slonim AD, Marcin JP, Pollack MM. Long-stay patients: Are there any long-term solutions? Crit Care Med 2003;31(1):313-314

3. Namachivayam P, Shann F, Shekerdemian L, et al. Three decades of pediatric intensive care: Who was admitted, what happened in intensive care, and what happened afterward. Pediatr Crit Care Med 2010;11(5):549-555. http://dx.doi.org/10.1097/PCC.0b013e3181ce7427

4. Briassoulis G, Filippou O, Natsi L, et al. Acute and chronic paediatric intensive care patients: Current 4. Briassoulis G, Filippou O, Natsi L, et al. Acute and chronic paediatric intensive care patients: Current
trends and perspectives on resource utilization. Q J Med 2004;97(8):507-518. http://dx.doi.org/10.1093/ qjmed/hch087

5. Conlon NP, Breatnach C, O'Hare BP, et al. Health-related quality of life after prolonged pediatric intensive care unit stay. Pediatr Crit Care Med 2009;10(1):41-44. http://dx.doi.org/10.1097/ PCC.0b013e31819371f6

6. Marcin JP, Slonim AD, Pollack MM, Ruttimann UE. Long-stay patients in the pediatric intensive care unit. Crit Care Med 2001;29(3):652-657.

7. Weissman C. Analyzing intensive care unit length of stay data: Problems and possible solutions. Crit Care Med 1997;25(9):1594-1600.

8. Van der Heide P, Hassing MB, Gemke RJ. Characteristics and outcome of long-stay patients in a paediatric intensive care unit: A case-control study. Acta Paediatr 2004:93(8):1070-1074. http://dx doi. org/ 10.1111/j.1651-2227.2004.tb02720.x

9. Brown KL, Ridout DA, Goldman AP, et al. Risk factors for long intensive care unit stay after cardiopulmonary bypass in children. Crit Care Med 2003;31(1):28-33.

10. Jeena PM, Wesley AG, Coovadia HM. Admission patterns and outcomes in a paediatric intensive care . Jeena PM, Wesley AG, Coovadia HM. Admission patterns and outcomes in a paediatric intensivec
unit in South Africa over a 25 -year period (1971-1995). Intensive Care Med 1999;25(1):88-94.

11. Naghib S, van der Starre C, Gischler SJ, et al. Mortality in very long-stay pediatric intensive care unit patients and incidence of withdrawal of treatment. Intensive Care Med 2010;36(1):131-136. http:// dx.doi.org/10.1007/s00134-009-1693-z

12. Kapil D, Bagga A. The profile and outcome of patients admitted to a pediatric intensive care unit. Indian J Pediatr 1993;60(1):5-10. http://dx.doi.org/10.1007/BF02860496

13. Solomon LJ, Morrow BM, Argent AC. Paediatric index of mortality scores: An evaluation of function in the paediatric intensive care unit of the Red Cross War Memorial Children's Hospital. South Afr J Crit Care 2014;30(1):8-13. http://www.sajcc.org.za/index.php/SAJCC/article/view/166/0 (accessed 5 December 2016).

14. Studdert DM, Burns JP, Mello MM, et al. Nature of conflict in the care of pediatric intensive care patients with prolonged stay. Pediatrics 2003;112(3):553-558.

15. Ebrahim S, Singh S, Hutchison JS, et al. Adaptive behavior, functional outcomes, and quality of life outcomes of children requiring urgent ICU admission. Pediatr Crit Care Med 2013;14(1):10-18. http:// outcomes of children requiring urgent ICU

16. Davydow DS, Richardson LP, Zatzick DF, Katon WJ. Psychiatric morbidity in pediatric critical illness Davydow DS, Richardson LP, Zatzick DF, Katon WJ. Psychiatric morbidity in pediatric critical illness
survivors: A comprehensive review of the literature. Arch Pediatr Adolesc Med 2010;164(4):377-385. survivors: A comprehensive review of the lite

17. Mayosi BM, Lawn JE, van Niekerk A, et al. Health in South Africa: Changes and challenges since 2009. Lancet 2012;380(9858):2029-2043. http://dx.doi.org/10.1016/S0140-6736(12)61814-5

Accepted 22 September 2016. 